\title{
A THEORY FOR THE SEPARATION OF LIQUID MIXTURES BY LASER RADIATION
}

\author{
H.G. HASSANOV* \\ Azerbaijan State Oil Research \& Project Institute (AzNSETLI), \\ Aga-Neymatullah St. 39, Baku 370033, Azerbaijan
}

(Received 26 September 2003; In final form 20 October 2003)

\begin{abstract}
A theory for the separation of liquid mixtures under the action of electromagnetic waves is developed. The theory considers physical specifications that take place during the separation. It is shown that at certain extreme value of the radiation intensity the maximum of mixture separation could be observed. This extreme value depends on the parameters of falling waves and medium studied. The effect of the liquid convection on the mixture separation is investigated. It is established that convection reduces the separation. Thus, laminar flow is much more preferable for the mixture separation than turbulent one.
\end{abstract}

Keywords: Liquid mixture separation; Electromagnetic waves; Radiation intensity; Convection flow; Opto-acoustic signals

\section{INTRODUCTION}

The possibility of separation of multi-phase mixtures by means of various physical fields is a very interesting problem from both theoretical and practical stand points. Some consideration has been given in recent paper [1], for example, it was noted in [2] that this external agent was proposed for determining the resin content in oils. Also, electromagnetic waves can be used to extract information about mixture fractions. As it has been established in [3], the form of the opto-acoustic signals in liquids depends strongly on the physical and rheological properties of the liquid (heat conductivity, heat capacity, viscosity, etc.). According to this fact, the application of electromagnetic waves (the laser radiation) may be used as precise and operative method for the fraction identification in mixtures. In addition to a brief review, one variant of the two-phase mixture separation by electromagnetic waves was also considered in [1]. In this article I shall develop further the ideas proposed in [1], in order to create the theory of liquid separation by means of high-frequency electromagnetic waves (laser radiation).

It is well accepted that the mechanism of separation is thermal. It is well known that under the action of laser radiation on any liquid some part of the beam energy

*E-mail: hhassanov@yahoo.com 
transforms of the internal energy of the liquid [4]. This leads to intensive heating of the liquid and all thermophysical properties of the liquid, including heat capacity, change sharply. It has been shown recently by Hassanov [5] that two jumps in the dependence of the heat capacity on the radiation intensity for a two-phase liquid, which correspond to appropriate phases, can be observed. It is proposed that this phenomenon means the separation of liquid mixture. One can evaluate the threshold value of the intensity $I_{\text {thr }}$ under which the separation can be realized. It follows from the formulae in [5] that the value of $I_{\mathrm{thr}}$ is estimated to be $\geq 10^{12} \mathrm{~W} / \mathrm{m}^{2}$.

\section{PROBLEM OF MIXTURE SEPARATION BY THE LASER RADIATION}

It is well known that the action of electromagnetic waves on liquid leads to the generation of opto-acoustic signals in the liquid [4]. The opto-acoustic mechanism of flow generation in liquid mixtures was first discussed in [6], and the separation was connected to periodic changes of pressure $P$ in the equation of state $\mu=\mu(c, P, T)$, where $\mu$ is the chemical potential, $c$ is the concentration and $T$ is the temperature.

However, when considering the action of the electromagnetic waves on a liquid it is necessary to take into account the value of the vector of the alternating electric field, so that the equation of state could be represented as $\mu=\mu(c, P, T, E)$. Moreover, as distinguished from the results of [6], a regime can be realized in which the electric field undergoes periodic changes. In other words, it is assumed that in liquid there is the electric wave:

$$
E=E_{0}+\tilde{E}(z) e^{i \omega t}
$$

where $E_{0}$ is the mean value of the medium electrification, $\omega$ is the frequency of the waves and $\tilde{E}(z)$ is the amplitude of the vibrations of the electric field, which depends on the coordinate.

Since most real liquids have magnetic permeability close to unity, the effect of the alternating magnetic field $H$ on the separation process will not be investigated here. Such a problem, but under the action of the temperature field, has been already considered in $[7,8]$.

Then, let us insert the expression for diffusion flow [7]:

$$
j=-\alpha \nabla \mu-\beta \nabla T
$$

into the continuity equation and follow the method developed in [9] ( $\alpha$ and $\beta$ are the kinetic coefficients that describe the diffusion properties of the medium [7]). As a result, after averaging by the period of the electric field vibration [1], one obtains an equation for the mean concentration change $\langle c\rangle$ (value of $\langle c\rangle$ represents the difference between the concentration of the initial liquid mixture and that of the newly separated phase):

$$
\frac{\partial\langle c\rangle}{\partial t}=D\left(\frac{\partial^{2}\langle c\rangle}{\partial z^{2}}+\frac{\alpha}{2 \rho D} \frac{\partial^{2} \mu}{\partial E^{2}} \frac{\partial^{2}\left\langle\tilde{E}^{2}\right\rangle}{\partial z^{2}}\right)
$$


where $D=(\alpha / \rho)(\partial \mu / \partial c)_{P, T}$ is the coefficient of the drift diffusion in the electric field and $\rho$ is the density of the liquid. Equation (2) shows that in the stationary regime, in a closed volume, the flow stimulated by the electric field will be compensated by the flow caused by 'usual' concentration diffusion (due to Fick's law). The solution of Eq. (2) gives a distributions $\langle c\rangle(z, t)$; in other words, dependence of the change of the mixture concentration by coordinate $z$ as a function of the intensity of the electric field. From a physics point of view, this magnitude represents the degree of mixture separation; the greater value of $\langle c\rangle$, the lower the separation of the mixture.

First, it is worthwhile to note that the value $\left\langle\tilde{E}^{2}\right\rangle$ is the intensity of the falling electromagnetic wave. In connection with this circumstance, Eq. (2) can be rewritten:

$$
\frac{\partial\langle c\rangle}{\partial t}=D\left(\frac{\partial^{2}\langle c\rangle}{\partial z^{2}}+K_{E}^{(0)} e^{-\delta z}\right)
$$

in which, as a result of the absorption by the Bouger's law, the reduction of the electromagnetic wave intensity within the liquid was realized, has already been taken into account.

$$
I(z)=I_{0} e^{-\delta z}
$$

where $\delta$ is the coefficient of studied medium absorption and $K_{E}^{(0)}$ is a constant equal to:

$$
K_{E}^{(0)}=\frac{\alpha \delta^{2}}{2 \rho D} \frac{\partial^{2} \mu}{\partial E^{2}} I_{0}
$$

Equation (3) should be solved under the following initial and boundary conditions

$$
\begin{gathered}
\langle c\rangle(z, t=0)=0 \\
\langle c\rangle(z=0, t)=c_{\infty}\left(1-e^{-\chi t}\right), \quad \frac{\partial\langle c\rangle(z=0, t)}{\partial z}=\phi
\end{gathered}
$$

In order to solve the problem of Eqs. (1)-(4) let us the Laplace transformation by time. After realizing the transformation, one has the following problem in the imaginary plane $(z, s)$ :

$$
s c^{*}=D \frac{d^{2} c^{*}}{d z^{2}}+\frac{K_{E}^{(0)}}{s} e^{-\delta z}, \quad c^{*}=c^{*}(z, s)=\int_{0}^{\infty}\langle c\rangle(z, t) e^{-s t} d t
$$

with boundary conditions:

$$
c^{*}(z=0, s)=c_{\infty} \frac{\chi}{s(s+\chi)}, \quad \frac{d c^{*}(z=0, s)}{d z}=\phi
$$

where $\phi$ is a negative permanent magnitude describing the diffusion properties of the liquid at the point $z=0$. 
The required function should be represented as:

$$
c^{*}(z, s)=F(z, s) e^{-\delta z}
$$

where $F(z, s)$ is a function, which must be determined.

Such an approach gives an opportunity to avoid the exponential factor. After realizing all mathematical calculations one gets the following equation relative to the function $F(z, s)$ :

$$
\frac{d^{2} F(z, s)}{d z^{2}}-2 \delta \frac{d F(z, s)}{d z}+\left(\delta^{2}-\frac{s}{D}\right) F(z, s)=-\frac{K_{E}^{(0)}}{s D}
$$

Equation (8) represents the forced vibration, its solution depends on the parameter $s$ of the Laplace transformation [1].

Using the results obtained in [1], for the stationary regime of the mixture separation, one can write:

$$
c^{*}(z, s)=C_{1} z+C_{2}+\frac{K_{E}^{(0)}}{s\left(s+D \delta^{2}\right)} e^{-\delta z}
$$

where constants $C_{1}$ and $C_{2}$ must be determined from Eq. (6).

After solving the system consisting of two equations relative to the unknown parameters $C_{1}$ and $C_{2}$, one obtains

$$
C_{1}=\frac{\phi}{s}+\frac{K_{E}^{(0)} \delta}{s\left(s+D \delta^{2}\right)}, \quad C_{2}=\frac{1}{s}\left\{c_{\infty} \frac{\chi}{s+\chi}-\frac{K_{E}^{(0)}}{s+D \delta^{2}}\right\}
$$

We can now finally write the function $c^{*}(z, s)$ as

$$
c^{*}(z, s)=\frac{1}{s}\left\{\phi+\frac{K_{E}^{(0)} \delta}{\left(s+D \delta^{2}\right)}\right\} z+\frac{1}{s}\left\{c_{\infty} \frac{\chi}{s+\chi}-\frac{K_{E}^{(0)}}{s+D \delta^{2}}\right\}+\frac{K_{E}^{(0)} \delta}{s\left(s+D \delta^{2}\right)} e^{-\delta z}
$$

Having returned to the real plane $(z, t)$ by the basic formulae of the operational calculus, one obtains:

$$
\langle c\rangle(z, t)=\left[\phi+K_{E}^{(0)}\left(\frac{1-e^{-D \delta t}}{D \delta}\right)\right] z-c_{\infty} \chi \frac{e^{-\chi t}-1}{\chi}+K_{E}^{(0)} \frac{e^{-D \delta^{2} t}-1}{D \delta^{2}}\left(1-e^{-\delta z}\right)
$$

We are interested in the stationary regime at $t \rightarrow \infty$, so one can neglect all summands with the exponential factor $\exp \left(-\beta_{i} t\right)$, where all $\beta_{i}$ are positive permanent magnitudes (see Eq. (12)). In this case the solution of Eq. (12) can be essentially simplified to the following form:

$$
\langle c\rangle(z, t)=A_{1}+A_{2} z+A_{3} e^{-\delta z}
$$


Correctly speaking, Eq. (13) describes the dependence of the mixture separation along the coordinate $z$ under the laser radiation with intensity $I_{0}$. The following abbreviations have been introduced in Eq. (13):

$$
A_{1}=c_{\infty}-\frac{K_{E}^{(0)}}{D \delta^{2}}, \quad A_{2}=\phi+\frac{K_{E}^{(0)}}{D \delta}, \quad A_{3}=\frac{K_{E}^{(0)}}{D \delta^{2}}
$$

It is clear from Eq. (13) that the mixture separation monotonically decreases with $z$. Nevertheless, special cases can be possible, in which the separation has to undergo the extremum. If the following inequality can be applied:

$$
\phi \prec \frac{K_{E}^{(0)}}{D \delta}=\frac{\alpha \delta}{2 \rho D^{2}} \frac{\partial^{2} \mu}{\partial E^{2}} I_{0}
$$

then one can observe maximum of the mixture separation at a distance $z_{\text {crit }}$, defined by

$$
z_{\text {crit }}=\frac{1}{\delta} \ln \left[\frac{K_{E}^{(0)}}{K_{E}^{(0)}-\phi D \delta}\right]
$$

Analysis of Eq. (13) shows that such an opportunity may be realized if parameters of the process are connected by the next formula:

$$
\frac{K_{E}^{(0)}}{K_{E}^{(0)}-\phi D \delta} \succ 1
$$

The last formula means that there is an extreme value of the intensity $I_{\text {ext }}$ of the falling electromagnetic waves, starting from which above mentioned maximum would be possible.

The calculations show that this value is defined by a relationship:

$$
I_{\text {ext }} \succ \frac{2 \rho \phi D^{2}}{\alpha\left(\partial^{2} \mu / \partial E^{2}\right)}
$$

and depends on physical and chemical properties of the liquid. In all other cases when there is a condition $I \leq I_{\text {ext }}$, one can observe only monotonous decrease in the mixture separation.

Some results of this theoretical investigation are shown on Fig. 1. For visualization the following parameters of the process of liquid separation have been used [5]: $K_{E}^{(0)} / D \delta^{2} \sim 5 \times 10^{20} \mathrm{~m}^{-3}, c_{\infty} \sim 2 \times 10^{20} \mathrm{~m}^{-3}, \delta \sim 5 \mathrm{~m}^{-1}$ (a typical value for transparent liquids) and three values of the concentration gradient $\phi \sim-(10,15,20) \times 10^{20} \mathrm{~m}^{-3}$.

In Fig. 1 Eq. (13) has been used for calculations. Curve 1 corresponds to $\phi \sim-10 \times 10^{20} \mathrm{~m}^{-3}$, curve 2 corresponds to $\phi \sim-15 \times 10^{20} \mathrm{~m}^{-3}$, curve $3-\phi \sim$ $-20 \times 10^{20} \mathrm{~m}^{-3}$. The curves has been calculated by the formulas: $\langle c\rangle(z)=-3+15 z+$ $5 e^{-5 z},\langle c\rangle(z)=-3+10 z+5 e^{-5 z},\langle c\rangle(z)=-3+5 z+5 e^{-5 z}$, respectively. 


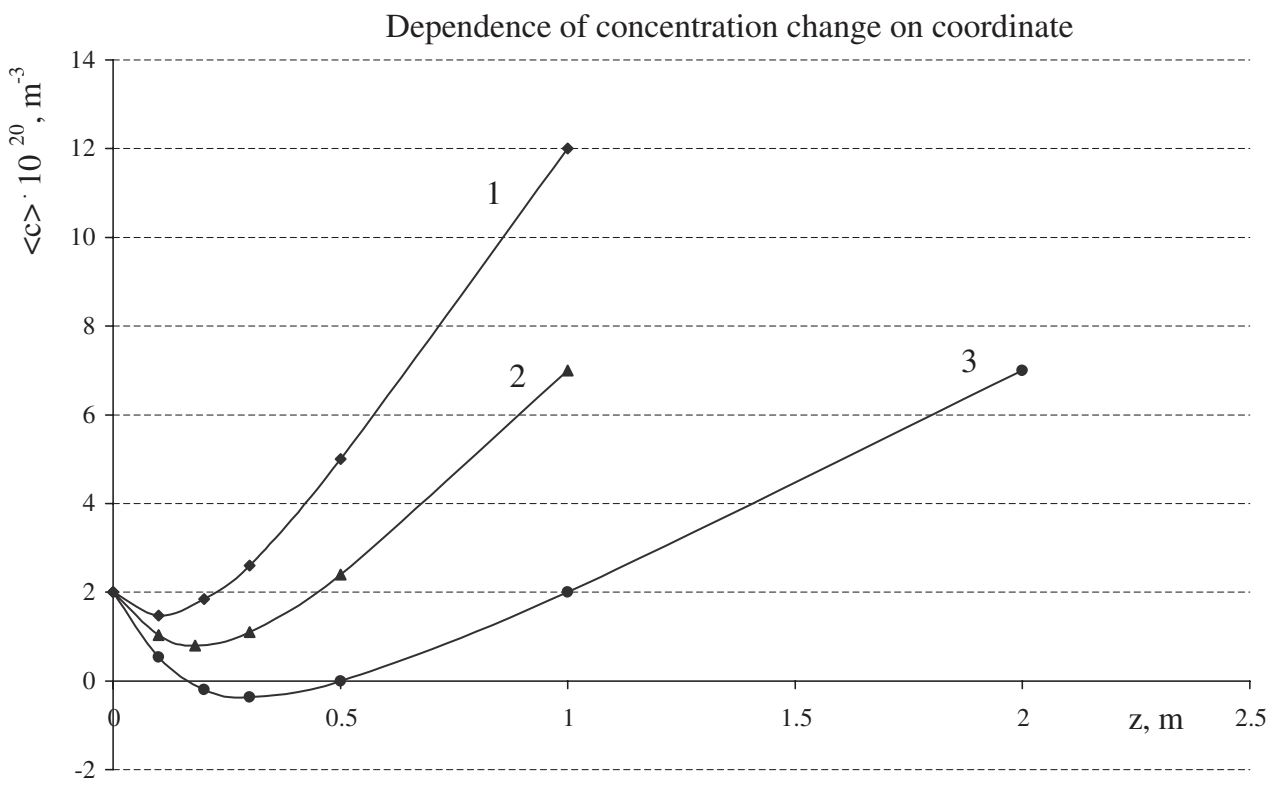

FIGURE 1 Dependence of concentration change on coordinate. Note: curves 1, 2, 3 correspond to various values of parameter $\phi$; curve $1: \phi \sim-10 \times 10^{20} \mathrm{~m}^{-3}$, curve $2: \phi \sim-15 \times 10^{20} \mathrm{~m}^{-3}$, curve $3: \phi \sim-20 \times 10^{20} \mathrm{~m}^{-3}$.

It can be concluded from the analysis of Fig. 1, that at the values of the concentration gradient $\phi \sim-(10,15) \times 10^{20} \mathrm{~m}^{-3}$ the partial separation of the liquid mixture will be observed for the entire interval of the coordinate $z$, with appropriate maxima. The partial separation takes place because the value of $\langle c\rangle$ does not reach zero, as can be seen in Fig. 1. However, for $\phi \sim-20 \times 10^{20} \mathrm{~m}^{-3}$ there is an area in which full separation of the liquid mixture can be observed. Negative values of $\langle c\rangle(z)$ mean that the newly created phase concentration exceed that of the initial liquid. This interval of the values $z$ runs between $0.17 \leq z \leq 0.53 \mathrm{~m}$; this interval may be considered as the separation scale for the problem involved (a typical tube length of $1 \mathrm{~m}$ has been used in experiments by opto-acoustic signals propagation in liquids [2]).

\section{EFFECT OF VARIOUS FACTORS ON SEPARATION}

The process of mixture separation is sensitive to the external conditions of the process, for example, to various physical fields, liquid convection, etc. It has been shown in [10] how an applied magnetic field can change displacement of the phases boundary. Furthermore, in [1] the author determined the influence of the form of chemical potential on mixture separation. In this section the problem of the effects of mixture convection on the separation process will be addressed.

It was shown by Galich [11] that for certain values of the laser radiation intensity $I^{*}=\lambda g / \alpha^{*} R c_{p}$ the convective laminar flow is stimulated in liquids (herein $\alpha^{*}$ is the coefficient of optical absorption, $R$ is the half-width of the falling laser beam while all other magnitudes are well defined [11]). With the increasing radiation intensity the laminar flow can be transformed into a turbulent one, and this transition can be realized at 
small values of the Reynolds number $R e \approx 25 \div 30$. Theoretical interpretation of this phenomenon is given in $[11,12]$ while the experiments confirming the early turbulence were described in [13]. My purpose is to find how the mixture convection affects the separation.

For this variant of problem, the equation of the diffusion, after averaging the concentration change, can be written as:

$$
\frac{\partial\langle c\rangle}{\partial t}+v \frac{\partial\langle c\rangle}{\partial z}=D\left(\frac{\partial^{2}\langle c\rangle}{\partial z^{2}}+\frac{\alpha}{2 \rho D} \frac{\partial^{2} \mu}{\partial E^{2}} \frac{\partial^{2}\left\langle\tilde{E}^{2}\right\rangle}{\partial z^{2}}\right)
$$

this equation must be solved under the same initial and boundary conditions given by Eq. (4). The algorithm of the solution for the problem is identical to that described in Section 2. After realizing the Laplace transformation, an equation in images is obtained:

$$
\frac{d^{2} F(z, s)}{d z^{2}}-\left(2 \delta+\frac{v}{D}\right) \frac{d F(z, s)}{d z}+\left(\delta^{2}-\frac{s}{D}+\frac{v \delta}{D}\right) F(z, s)=-\frac{K_{E}^{(0)}}{s D}
$$

The form of the solution of Eq. (17) depends on parameter $\sigma$, which is determined by [14]:

$$
\sigma=\sqrt{\left(\frac{v}{D}\right)^{2}+4 \frac{s}{D}}
$$

I again consider a stationary regime of separation, that is $t \rightarrow \infty$ (in images, this approximation corresponds to $s \rightarrow 0$ ). Then, from Eq. (17) it can be written:

$$
F(z, s)=C_{1} e^{\delta z}+C_{2} e^{(\delta+(v / D)) z}
$$

and the required solution [14]:

$$
c^{*}(z, s)=C_{1}+C_{2} e^{(v / D) z}+\frac{K_{E}^{(0)}}{s\left(s+D \delta^{2}\right)} e^{-\delta z}
$$

By determining the constant $C_{1}$ and $C_{2}$ from the boundary conditions of Eq. (6), the functions $c^{*}(z, s)$ and $\langle c\rangle(z, t)$ can thus be determined.

However, preliminary analysis of Eq. (19) gives an opportunity to conclude that the mixture convection reduces the separation. In the first approximation of Eq. (19) one can expand the exponential factor $\exp ((v / D) z)$ into the series and take into consideration the first two summands:

$$
\exp \left(\frac{v}{D} z\right) \approx 1+\frac{v}{D} z
$$


Then, after some mathematical calculations one obtains:

$$
\langle c\rangle(z, t)=A_{1}+A_{2}^{*} z+A_{3} e^{-\delta z}
$$

in which $A_{2}^{*}=(D / v) A_{2}$, that is the function $\langle c\rangle(z, t)$, is inversely proportional to the convection speed $v$ (compare this relation with Eq. (13)). Hence, during the mixture convection with speed $v$, the function $\langle c\rangle(z, t)$ will be lower under otherwise equal conditions. The last aspect means that the mixture separation without convection will be more efficient than for moving liquid.

\section{CONCLUSION}

This article has established some specifications relevant to mixture separation by laser radiation. Distribution of the concentration change $\langle c\rangle(z, t)$, which describes the mixture separation under the radiation, is defined as a result of two conflicting summands:

$$
\langle c\rangle(z, t)=A_{1}+A_{2} z+A_{3} e^{-\delta z}
$$

The first term, $\left(A_{2} z\right)$ represents separation due to the diffusion by the Fick's law while the second term $\left(A_{3} e^{-\delta z}\right)$ describes the absorption by the Bouger's law. In accordance with these terms the mixture separation exhibits a maximum, position of which depends on the radiation characteristics and thermophysical properties of the liquid. Furthermore, in practice two variants of separation can take place: full and partial. If the value of $\langle c\rangle$ can reach zero, then it can be concluded that full separation was realized. This variant is possible under a certain combination of the liquid thermophysical properties and the radiation characteristics (this deduction follows from the analysis of Eq. (13)).

However, if the values of $\langle c\rangle$ cannot reach zero, then the partial separation takes place in the liquid. It is worthwhile to mention that the separation will be realized up such values $z$, unless due to the Bouger's law, the radiation intensity reduces to threshold value $I_{\mathrm{thr}}$, as has been discussed in Section 1.

As has been noted above, separation of multi-component liquids (mixtures, solutions, emulsions, etc.) by means of electromagnetic waves can be used for practical purposes. However, the use of high-frequency electromagnetic waves is not limited to mixture separation. As has been theoretically analysed in [5], laser radiation can be applied for remote mixing of two different phases. This new method is based on the fact that two different liquid phases have different surface tension profiles. Furthermore, at some threshold value of the radiation intensity $I_{\mathrm{thr}}^{*}$ [5] these two phases begin to mix with each other, the value of which is connected with mutual permeation of their profiles.

Thus, it can be concluded that in practice high-frequency electromagnetic waves can be used for both the mixture separation, and two different phases mixing. It is obvious, that the laser radiation intensity is the basic characteristics controlling both processes. By means of the last parameter one can vary the speed and character of any relevant technological processes in which separation (and/or mixing) is important. 


\section{Acknowledgment}

The author would like to express his kind regards to Prof. G.T. Hassanov, Head of Physics Division, Azerbaijan State Oil Academy, with whom some parts of this article has been discussed.

\section{References}

[1] H.G. Hassanov and A.A. Aliyev, About liquid mixture separation under electromagnetic waves action, Journal Bilgi-Knowledge (Technical Series), 4 (2001), 9-14

[2] G.T. Hassanov and M.A. Musayev, Definition of resin-content of oil by laser opto-acoustic method, Proc. of Thermophysical Conference of CIS, Mahachkala, Russia, 1992, 85-88.

[3] G.T. Hassanov, A.A. Aliyev, L.P. Guryanova, About some physical properties of oils, Proc. of Inst. of Physics, 6 (2000), 4-7.

[4] V.E. Gusev and A.A. Karabutov, Laser Optoacoustics, Moskva, Nauka, 1991.

[5] H.G. Hassanov, Some Problems of Improvement of Technological Processes by Heat and Mass Transfer of Liquids, Baku, Khazar University Press, 2003.

[6] F. Kh. Gelmukhanov and G.G. Telegin, Laser separation of components of gas mixture which is in constant electric field, J. Tech. Phys., 55 (1985), 1559-1565.

[7] L.D. Landau and E.M. Lifshits, Hydrodynamics, Moskva, Nauka, 1986.

[8] A.M. Dykhne and A.N. Starostin, The theory of drift motion of molecules in field of resonance infrared radiation, J. Exper. Theor. Phys., 79 (1980), 1211-1228.

[9] A.S. Aloyev, A.C. Bakhtadze, V.P. Kovtun, A.V. Chanturiya, About separation of liquid mixtures in the field of temperature wave, J. Tech. Phys., 58 (1988), 1794-1796.

[10] H.G. Hassanov, A mathematical model for the separation of viscous liquid by magnetic field, J. Math. Comp. App., 8 (2003), 13-19.

[11] N.E. Galich, Turbulence generated by laser radiation in standing and moving gas (liquid), J. Tech. Phys., 50 (1980), 1196-1202.

[12] H.G. Hassanov, Hydrodynamic Research of Interaction Between Acoustic and Laser Radiations and Liquid, Open World, Baku, 2002.

[13] V.I. Zuyev, Experimental investigation of convection caused by laser radiation, J. Tech. Phys., 56 (1986), 394-396.

[14] E. Kamke, Handbook on Ordinary Differential Equations, Moskva, Nauka, 1976.

\section{BIOGRAPHY}

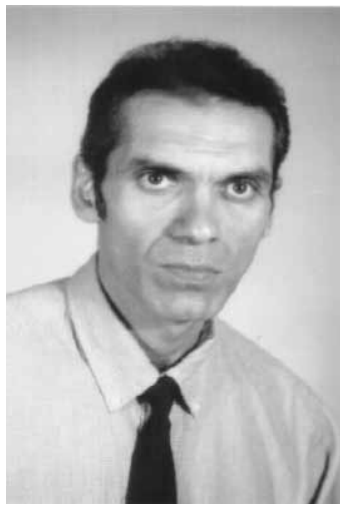

Hikmet Hassanov graduated from the Azerbaijan State University in Baku, Azerbaijan, in 1984. Until 1996 he was associated with the Institute of Physics, of the Academy of Sciences in Baku. In 1992 he obtained his Ph.D. In 1996 he joined the Azerbaijan State Oil Research \& Project Institute (AzNSETLI), Ministry of Fuels and Energetics in Baku as a senior scientific researcher. Dr. Hassanov's areas of interest are mechanics of fluids, heat and mass transfer, hydrodynamics and the effects of physical fields on properties of fluids. He published more than 50 papers and he is a member of the Scientific Council of AzNSETLI. Since 1997 he also associated with the Khazar University in Baku as a visiting lecturer. 